\title{
EXTENDING CR FUNCTIONS FROM MANIFOLDS WITH BOUNDARIES
}

\section{A. Tumanov}

\section{Introduction}

The extendibility of $\mathrm{CR}$ functions on smooth real manifolds in $\mathbf{C}^{N}$ has been extensively studied by many specialists (see, e.g., [Bo] for references). In this paper we generalize the results of [T1] and [T2] on the extendibility of CR functions to manifolds with boundaries and edges.

Recall that a generic manifold $M \subset \mathbf{C}^{N}$ is said to be minimal at $p \in M$ if there is no proper CR submanifold $S \subset M$ passing through $p$ with the same $\mathrm{CR}$ dimension as $M$. According to [T1], if $M$ is minimal at $p$, then all $\mathrm{CR}$ functions on $M$ extend to the same full dimensional wedge with edge $M$ near $p$. Conversely, Baouendi and Rothschild [BR] show that if $M$ is not minimal at $p$, then there are $\mathrm{CR}$ functions in a neighborhood of $p$ on $M$ that do not extend to any full dimensional wedge with edge $M$. Nevertheless, all CR functions on $M$ generally still extend to a larger manifold [BP] [T2]. This manifold has the form of a wedge of dimension lower than the dimension of the ambient space. We call it a manifold with edge $M$, reserving the term wedge for full dimensional sets in $\mathbf{C}^{N}$. Since the natural domains of extended CR functions are manifolds with edges, it is appropriate to consider CR functions initially defined on such sets.

The simplest case of a manifold with edge is a manifold with boundary. Let $M \subset \mathbf{C}^{N}$ be a manifold with generic boundary $M_{0}$. Suppose $M$ is minimal at all interior points. Then by [T1] all CR functions on $M$ extend over a wedge $W$ with edge $M$. A natural question is the boundary behavior of this wedge at $M_{0}$. We show that $W$ approaches $M_{0}$ as a wedge with edge $M_{0}$ (Corollary 1.3).

In the general case, we introduce the defect of a manifold $M$ with edge $M_{0}$ at a point $p \in M_{0}$ and show that unless the defect is maximal, all CR

Received April 26, 1995.

Supported by NSF Grant DMS-9401652. 
functions on $M$ extend to a larger manifold $W$ with the same edge $M_{0}$. The dimension of $W$ is related to the defect of $M$ at $p$. In particular, we introduce the notion of a minimal point on the edge of the manifold. If $M$ is minimal at $p \in M_{0}$, we show that all CR functions on $M$ extend over a full dimensional wedge with edge $M_{0}$ near $p$ (Corollary 1.2).

The paper is organized as follows. In Section 1 we formulate the main result and some consequences. Section 2 includes some preliminaries. In Sections 3 and 4 we prove the main result. The exposition in this paper is self-contained except that we refer to [T1] for the proof of technical Lemma 2.3.

\section{Main results}

Let $M$ be a smooth real manifold in $\mathbf{C}^{N}$. Let $T_{p}^{c}(M)=T_{p}(M) \cap J T_{p}(M)$ be the maximal complex subspace of the tangent space $T_{p}(M)$ at a point $p \in M$, where $J$ is the operator of multiplication by the imaginary unit in $\mathbf{C}^{N}$. Recall that the manifold $M$ is called a $C R$ manifold if all the spaces $T_{p}^{c}(M), p \in M$, have the same dimension. This dimension is called the $C R$ dimension of $M$ and denoted by $\mathrm{CR} \operatorname{dim}(M)$ here. Recall that a manifold $M$ is called generic if $T_{p}(M)+J T_{p}(M)=T_{p}\left(\mathbf{C}^{N}\right) \simeq \mathbf{C}^{N}, p \in M$. A generic manifold $M \subset \mathbf{C}^{N}$ is always a $\mathrm{CR}$ manifold. We denote the real codimension of $M$ in $\mathbf{C}^{N}$ by $\operatorname{codim}(M)=2 N-\operatorname{dim}(M)$. For a generic manifold $M \subset \mathbf{C}^{N}$, we have $\operatorname{CRdim}(M)+\operatorname{codim}(M)=N$.

Recall that a smooth, complex valued function on $M$ is called a $C R$ function if its differential is $\mathbf{C}$-linear on $T^{c}(M)$. A continuous function is called a CR function if the last condition holds in the sense of distribution theory. We denote by $\mathrm{CR}(M)$ the set of all continuous $\mathrm{CR}$ functions on $M$.

Let $M$ and $M_{0}$ be manifolds in $\mathbf{R}^{n}$, and $M_{0} \subset \bar{M}$. We call $M$ a manifold with edge $M_{0}$, if for any point $p \in M_{0}$, there exist open sets $\Omega \subset \mathbf{R}^{n}$, $U \subset M_{0} \times \mathbf{R}^{l}$ (where $l=\operatorname{dim}(M)-\operatorname{dim}\left(M_{0}\right)$ ), an open convex cone $\Gamma \subset \mathbf{R}^{l}$, and a smooth embedding $F: U \rightarrow \mathbf{R}^{n}$ such that $p \in \Omega,(p, 0) \in U$, $\Omega \cap M=F\left(U \cap\left(M_{0} \times \Gamma\right)\right)$. Since we are interested in local questions, we assume that $\Gamma$ does not depend on $p$.

For $l=1, M$ is a manifold with boundary $M_{0}$. For $l=n-\operatorname{dim}\left(M_{0}\right)$, $M$ is a full-dimensional wedge with edge $M_{0}$.

Let $C^{k, \alpha}$ denote the space of functions with derivatives up to order $k$ satisfying a Lipschitz condition with exponent $\alpha$. We say that the manifold $M$ with edge $M_{0}$ is $C^{k, \alpha}$ smooth (where $k \geq 1$ and $0<\alpha<1$ ) if the defining mapping $F$ belongs to $C^{k, \alpha}$. 
As we mentioned in the introduction, we consider CR functions defined on manifolds with edges. We say that $f$ is a $\mathrm{CR}$ function on a CR manifold $M$ with edge $M_{0}$ and write $f \in \mathrm{CR}\left(M \cup M_{0}\right)$ if $f \in C\left(M \cup M_{0}\right) \cap \mathrm{CR}(M)$.

The substance of this paper is the following theorem.

Theorem 1.1. Let $M \subset \mathbf{C}^{N}$ be a $C^{k, \alpha}$ smooth generic manifold with generic edge $M_{0}$, where $k \geq 2$ and $0<\alpha<1$. Then for every point $p \in M_{0}$, there exists an integer $d=\operatorname{def}(p)=\operatorname{def}\left(p, M_{0}, M\right), 0 \leq d \leq \operatorname{codim}(M)$, called the defect of $M$ at $p$ such that the following two statements (A) and (B) hold:

(A) $\exists$ a manifold $W$ with edge $M_{0}$ such that $\operatorname{dim}(W)=2 N-d$ and all $C R$ functions on $M \cup M_{0}$ extend to be $C R$ on $W \cup M_{0}$. The manifold $W$ has almost the same Lipschitz smoothness as $M$. Precisely, for every $0<\beta<\alpha$, there exists a neighborhood $U \subset \mathbf{C}^{N}$ of $p$ such that $W \cap U$ is of class $C^{k, \beta}$.

(B) $\exists$ a neighborhood $U \subset \mathbf{C}^{N}$ of $p$ and a closed $C R$ submanifold $S \subset$ $M \cap U$ with edge $S_{0}=M_{0} \cap \bar{S}$ such that $p \in S_{0}, \operatorname{dim}(S)=\operatorname{dim}(M)-d$ and $\mathrm{CRdim}(S)=\mathrm{CRdim}(M)$. The manifold $S$ is $C^{k, \beta}$-smooth for all $0<\beta<\alpha$.

Note.

(1) The assumption that $M$ is generic can be dropped here. Indeed, since $M_{0}$ is generic, $M$ is automatically generic near $M_{0}$.

(2) $M_{0}$ is of class $C^{k, \alpha}$ (by our definition of the manifold with edge of class $C^{k, \alpha}$ ).

(3) The manifold $M_{0}$ in Theorem 1.1 may be totally real, in which case $\operatorname{CRdim}\left(M_{0}\right)=0$. On the contrary, $\operatorname{CRdim}(M) \geq 1$ because $M_{0}$ is generic.

(4) The conditions $\left(\mathrm{CRdim}(S)=\mathrm{CRdim}(M)\right.$ and $M_{0}$ is generic) imply that $\bar{S}$ is transversal to $M_{0}$ in $\bar{M}$. Therefore, $S_{0}=M_{0} \cap \bar{S}$ is automatically a smooth manifold, $\operatorname{dim}\left(M_{0}\right)-\operatorname{dim}\left(S_{0}\right)=\operatorname{dim}(M)-$ $\operatorname{dim}(S)$ and $\mathrm{CRdim}\left(S_{0}\right)=\mathrm{CRdim}\left(M_{0}\right)$. In particular, if $M$ is a manifold with boundary $M_{0}$, then $S$ is a manifold with boundary $S_{0}$.

Our Theorem 1.1 is a direct generalization of the results of [T1] and [T2] on the extendibility of CR functions from manifolds without edges. Recall that a CR manifold $M \subset \mathbf{C}^{N}$ is minimal at a point $p \in M$ if there is no CR submanifold $S \subset M$ such that $p \in S, \operatorname{CRdim}(S)=\operatorname{CRdim}(M)$, 
but $\operatorname{dim}(S)<\operatorname{dim}(M)$ [T1]. Likewise, we say that a manifold $M$ with edge $M_{0}$ is minimal at $p \in M_{0}$ if there is no closed submanifold $S \subset M$ in a neighborhood of $p$ such that $p \in \bar{S}, \operatorname{CRdim}(S)=\operatorname{CRdim}(M)$, but $\operatorname{dim}(S)<\operatorname{dim}(M)$.

Corollary 1.2. Let $M$ be a manifold with the generic edge $M_{0}$. Assume that $M$ is minimal at $p \in S_{0}$. Then all $C R$ functions on $M \cup M_{0}$ extend to be holomorphic in the same full-dimensional wedge with edge $M_{0}$ near $p$.

By (A), $d=\operatorname{def}(p)>0$ if CR functions on $M \cup M_{0}$ do not extend into any full-dimensional wedge of edge $M_{0}$ near $p \in M_{0}$. Then (B) contradicts the assumption that $M$ is minimal at $p$. We note that Corollary 1.2 gives new information only if the edge $M_{0}$ itself is not minimal at $p$. For instance, this is the case when $M_{0}$ is totally real.

If the manifold $M$ with edge $M_{0}$ is minimal at all interior points, it is certainly minimal as a manifold with edge whence the following:

Corollary 1.3. Let $M$ be a manifold with the generic edge $M_{0}$. Assume that $M$ is minimal at all interior points. Then all $C R$ functions on $M \cup M_{0}$ extend to be holomorphic in the same full-dimensional wedge with edge $M_{0}$.

We give another consequence of the main theorem that relates the existence of submanifolds $S_{0} \subset M_{0}$ such that $\mathrm{CRdim}\left(S_{0}\right)=\mathrm{CRdim}\left(M_{0}\right)$, and the extendibility of $\mathrm{CR}$ functions defined on the manifold (without edge) $M_{0}$.

Corollary 1.4. Let $M_{0}$ be a generic manifold in $\mathbf{C}^{N}$ and $p \in M_{0}$. Assume there is no CR submanifold $S_{0} \subset M_{0}$ such that $p \in M_{0}$,

$$
\begin{gathered}
\operatorname{CRdim}\left(S_{0}\right)=\mathrm{CRdim}\left(M_{0}\right) \quad \text { and } \\
\operatorname{dim}\left(M_{0}\right)-d^{\prime}<\operatorname{dim}\left(S_{0}\right)<\operatorname{dim}\left(M_{0}\right)-d^{\prime \prime},
\end{gathered}
$$

where $0 \leq d^{\prime \prime}<d^{\prime} \leq \operatorname{codim}\left(M_{0}\right)$. Then if a $C R$ function $f$ on $M_{0}$ extends to be $C R$ on a manifold $M^{\prime}$ with edge $M_{0}$ such that $p \in \bar{M}^{\prime}$ and $\operatorname{dim}\left(M^{\prime}\right)>$ $2 N-d^{\prime}$, then $f$ automatically extends to be $C R$ on another manifold $M^{\prime \prime}$ with edge $M_{0}$ such that $p \in \bar{M}^{\prime \prime}$ and $\operatorname{dim}\left(M^{\prime \prime}\right)=2 N-d^{\prime \prime}$.

This corollary gives new information only if there is a submanifold $S_{0} \subset$ $M_{0}$ such that $p \in S_{0}, \mathrm{CRdim}\left(S_{0}\right)=\mathrm{CR} \operatorname{dim}\left(M_{0}\right), \operatorname{dim}\left(S_{0}\right)<\operatorname{dim}\left(M_{0}\right)-d^{\prime \prime}$ (or $\operatorname{dim}\left(S_{0}\right) \leq \operatorname{dim}\left(M_{0}\right)-d^{\prime}$, which is the same). Otherwise, the conclusion of the corollary follows immediately from the result of [T2] on the extendibility of CR functions on manifolds without edges. In the special 
case in which $d^{\prime}=\operatorname{codim}\left(M_{0}\right)$ and $d^{\prime \prime}=0$, any manifold $S_{0} \subset M_{0}$ with $\mathrm{CRdim}\left(S_{0}\right)=\mathrm{CR} \operatorname{dim}\left(M_{0}\right)$ must be complex. The corollary then asserts that if a $\mathrm{CR}$ function $f$ on $M_{0}$ extends to be $\mathrm{CR}$ on a manifold $M$ with boundary $M_{0}$, then $f$ extends to be holomorphic in a full-dimensional wedge $W$ of edge $M_{0}$. It is of interest to find out if the tangent cone to $W$ depends on the direction of $M$ in the normal space of $M_{0}$, i.e., $T_{p}(M) / T_{p}\left(M_{0}\right)$.

We note that Corollary 1.4 is related to a result of [T3] (Theorem 6.1). In that theorem, instead of nonexistence of $S_{0}$ with the indicated properties, it is assumed that the connection that governs the propagation of extendibility of CR functions has sufficiently rich holonomy.

Proof of Corollary 1.4. By statement (A), it suffices to show that $d=$ $\operatorname{def}\left(p, M_{0}, M^{\prime}\right) \leq d^{\prime \prime}$. By statement (B), there is a submanifold $S \subset M^{\prime}$ with edge $S_{0} \subset M_{0}$ such that $p \in S_{0}, \operatorname{CRdim}(S)=\mathrm{CRdim}\left(M^{\prime}\right), \operatorname{dim}(S)=$ $\operatorname{dim}\left(M^{\prime}\right)-d$. Then $\operatorname{dim}(S) \geq 2 \mathrm{CR} \operatorname{dim}(S)=2 \mathrm{CR} \operatorname{dim}\left(M^{\prime}\right)=2\left(\operatorname{dim}\left(M^{\prime}\right)-\right.$ $N)$. Hence $d \leq 2 N-\operatorname{dim}\left(M^{\prime}\right)$. Using $\operatorname{dim}\left(M^{\prime}\right)>2 N-d^{\prime}$, we get $d<d^{\prime}$. The edge $S_{0}$ has dimension $\operatorname{dim}\left(S_{0}\right)=\operatorname{dim}\left(M_{0}\right)-d>\operatorname{dim}\left(M_{0}\right)-d^{\prime}$. Therefore, by the assumptions of the corollary, $\operatorname{dim}\left(S_{0}\right) \geq \operatorname{dim}\left(M_{0}\right)-d^{\prime \prime}$, whence $d \leq d^{\prime \prime}$.

\section{Analytic discs and their deformations}

Let $M \subset \mathbf{C}^{N}$ be a generic manifold with generic edge $M_{0}$. Assume that $M$ and $M_{0}$ are $C^{k, \alpha}$-smooth, where $k \geq 1,0<\alpha<1$.

An analytic disc in $\mathbf{C}^{N}$ is a continuous mapping $A: \bar{\Delta} \rightarrow \mathbf{C}^{N}$ holomorphic in the unit disc $\Delta$. We say that $A$ is attached to $M \cup M_{0}$ if $A(b \Delta) \subset M \cup M_{0}$.

Let $p \in M_{0}$. Let $\mathcal{A}_{p}$ be the set of all small $C^{k, \alpha}$-smooth discs attached to $M \cup M_{0}$ passing through $p$, that is

$\mathcal{A}_{p}=\left\{A \in \mathcal{O}(\Delta) \cap C^{k, \alpha}(\bar{\Delta}):\|A\|_{C^{k, \alpha}} \leq r_{0}, A(1)=p, A(b \Delta) \subset M \cup M_{0}\right\}$,

where $\mathcal{O}(\Delta)$ denotes all holomorphic functions in $\Delta$, and $r_{0}>0$ is small.

For a disc $(\zeta \mapsto A(\zeta)) \in \mathcal{A}_{p}$, the vector $-\partial A(1) / \partial \zeta$ determines the direction of the $\operatorname{disc} A$ at $p$. Let $V_{p}^{\prime}$ be the vector subspace of $T_{p}\left(\mathbf{C}^{N}\right) / T_{p}\left(M_{0}\right)$ spanned by the above direction vectors, that is

$$
V_{p}^{\prime}=\operatorname{Span}\left\{-\partial A(1) / \partial \zeta \bmod T_{p}\left(M_{0}\right): A \in \mathcal{A}_{p}\right\}
$$


Proposition 2.1. Let $\operatorname{dim}\left(V_{p}^{\prime}\right)=r$. Then there is a manifold $W$ with edge $M_{0}$ of dimension $\operatorname{dim}\left(M_{0}\right)+r$ such that all continuous $C R$ functions on $M \cup M_{0}$ extend to be $C R$ on $W$. For every $0<\beta<\alpha$, there exists a neighborhood $U \subset \mathbf{C}^{N}$ of $p$ such that $W \cap U$ is of class $C^{k, \beta}$.

Proof. The proof is a combination of the approximation theorem by Baouendi and Treves $[\mathrm{BT}]$, the edge-of-the-wedge theorem by Ayrapetian and Henkin [A], and a simple fact on the deformation of discs [T3]. Indeed, let $A_{1}, \ldots, A_{r} \in \mathcal{A}_{p}$ be the discs such that $v_{j}=-\partial A_{j}(1) / \partial \zeta(1 \leq j \leq r)$ span $V_{p}^{\prime}$. By deforming these discs, one obtains manifolds $M_{1}, \ldots, M_{r}$ with the same boundary $M_{0}$ such that each $T_{p}\left(M_{j}\right)$ is spanned by $T_{p}\left(M_{0}\right)$ and $v_{j}$. (See [T3, Proposition 1.3] for details.) Therefore $\operatorname{dim}\left(\sum_{j} T_{p}\left(M_{j}\right)\right)=$ $\operatorname{dim}\left(M_{0}\right)+r$. Since each $M_{j}$ is a union of discs attached to $M \cup M_{0}$, by the Baouendi-Treves approximation theorem, all CR functions on $M \cup M_{0}$ extend to be CR on $M_{j}$. The proposition now follows by the edge-of-thewedge theorem.

Remark. In our statement regarding the smoothness of $W$, the neighborhood $U$ depends on $\beta$. This has happened because of the repeated use of Bishop's equation. We use it for the first time to construct $M_{j}$-s. By the result of [T4] on the regularity of Bishop's equation, the manifolds $M_{j \text {-s }}$ are of class $C^{k, \beta}$ for all $0<\beta<\alpha$. However, the $C^{k, \beta}$ norms of defining functions of $M_{j}$-s blow up as $\beta \rightarrow \alpha$. We use Bishop's equations one more time to construct $W$ to be a union of discs attached to $\bigcup M_{j}$ (see $[\mathrm{A}]$ ). Applying the result of [T4] in this situation yields shrinking $U$ as $\beta \rightarrow \alpha$. The author does not know whether it is possible to choose $U$ independent of $\beta$.

We will use the Bishop equation $[\mathrm{B}]$ to describe the set $\mathcal{A}_{p}$. In a suitable holomorphic local system of coordinates $\left(z=x+i y \in \mathbf{C}^{m}, w \in \mathbf{C}^{n}\right)$ in $\Omega \subset \mathbf{C}^{N}$ with origin at $p=0$, the manifolds $M$ and $M_{0}$ can be defined as

$$
\begin{aligned}
M_{0} \cap \Omega & =\{(x+i y, w): x=h(y, w, 0)\}, \\
M \cap \Omega & =\{(x+i y, w): x=h(y, w, t), t \in \Gamma\} .
\end{aligned}
$$

where $h$ is a smooth real $\mathbf{R}^{m}$-valued function in a neighborhood of zero in $\mathbf{R}^{m} \times \mathbf{C}^{n} \times \mathbf{R}^{l}$ such that $h$ and the partial derivatives $h_{y}$ and $h_{w}$ vanish at zero while $h_{t}$ has the maximum rank $l$ at zero, that is

$$
h(0)=0, \quad h_{y}(0)=0, \quad h_{w}(0)=0, \quad \operatorname{rank} h_{t}(0)=l
$$


and $\Gamma \subset \mathbf{R}^{l}$ is an open convex cone. In this notation, $\operatorname{CRdim}\left(M_{0}\right)=n$, $\operatorname{codim}\left(M_{0}\right)=m$ and $\operatorname{dim}(M)=\operatorname{dim}\left(M_{0}\right)+l$.

Along with $M$, we consider $\tilde{M}$, the continuation of $M$ across the edge $M_{0}$, obtained by dropping the condition that $t \in \Gamma$ in $(2.1)$, that is

$$
\tilde{M}=\left\{(x+i y, w) \in \Omega: x=h(y, w, t), t \in \mathbf{R}^{l}\right\} .
$$

Let $q=(x+i y, w) \in \tilde{M}$. Then there is a unique $t \in \mathbf{R}^{l}$ such that $x=$ $h(y, w, t)$. We call such $t$ the $t$-component of $q$.

We denote by $\tilde{\mathcal{A}}_{p}$ the set of all small analytic $\operatorname{discs} A$ attached to $\tilde{M}$ with $A(1)=0$.

Proposition 2.2. There exists a unique disc $A \in \tilde{\mathcal{A}}_{p}$ with given sufficiently small $w$ - and t-components respectively $(\zeta \mapsto w(\zeta)) \in C^{k, \alpha}(\bar{\Delta}) \cap$ $\mathcal{O}(\Delta)$ and $(\zeta \mapsto \tau(\zeta)) \in C^{k, \alpha}(b \Delta)$ such that $w(1)=0, \tau(1)=0$. Furthermore, $A \in \mathcal{A}_{p}$ (2.1) if and only if $\tau(\zeta) \in \Gamma \cup 0$ for $\zeta \in b \Delta$.

Proof. Let $T_{1}$ denote the harmonic conjugation operator on $b \Delta$ normalized by the condition $\left(T_{1} \phi\right)(1)=0$. That is $T_{1} \phi=T \phi-(T \phi)(1)$ where $T$ is the standard Hilbert transform.

Let $A(\zeta)=(x(\zeta)+i y(\zeta), w(\zeta))$ and let $\zeta \mapsto \tau(\zeta)$ be the $t$-component of $\left.A\right|_{b \Delta}$, that is $x(\zeta)=h(y(\zeta), w(\zeta), \tau(\zeta))$ for $\zeta \in b \Delta$. Since the $x$ - and $y$-components of $A$ are harmonic conjugates, and $y(1)=0$, the function $\zeta \mapsto y(\zeta)$ satisfies the Bishop equation

$$
y=T_{1} h(y, w, \tau)
$$

For given $\zeta \mapsto w(\zeta)$ and $\zeta \mapsto \tau(\zeta)$ small in the $C^{k, \alpha}$ norm, this equation has a unique solution $\zeta \mapsto y(\zeta)$ in $C^{k, \alpha}$ (see [T4]). Once (2.5) is solved, $\zeta \mapsto x(\zeta)+i y(\zeta)$ is obtained by harmonically extending to $\Delta$. The characterization of $\mathcal{A}_{p}$ by the condition $\tau(\zeta) \in \Gamma \cup 0$ is obvious.

Proposition 2.2 identifies $\tilde{\mathcal{A}}_{p}$ with a neighborhood of zero in the Banach space

$$
\begin{aligned}
& \tilde{\mathcal{B}}=\{(w, \tau): \\
& \left.w \in C^{k, \alpha}\left(\bar{\Delta}, \mathbf{C}^{n}\right) \cap \mathcal{O}(\Delta), \tau \in C^{k, \alpha}\left(b \Delta, \mathbf{R}^{l}\right), w(1)=0, \tau(1)=0\right\},
\end{aligned}
$$

and $\mathcal{A}_{p}$ is represented by elements of

$$
\mathcal{B}=\{(w, \tau) \in \tilde{\mathcal{B}}: \tau(\zeta) \in \Gamma \cup 0 \text { for } \zeta \in b \Delta\}
$$


We write $A \leftrightarrow(w, \tau)$ if $w$ and $\tau$ are the $w$-and $t$-components of $A$.

A (infinitesimal tangential to $\tilde{M}$ ) deformation of a disc $A \in \tilde{\mathcal{A}}_{p}$ is a continuous mapping $\dot{A}: \bar{\Delta} \rightarrow \mathbf{C}^{N}$ holomorphic in $\Delta$ such that $\dot{A}(\zeta)=$ $(\dot{x}(\zeta)+i \dot{y}(\zeta), \dot{w}(\zeta)) \in T_{A(\zeta)}(\tilde{M})$ for $\zeta \in b \Delta$ and $\dot{A}(1)=0$.

Let $v=(\dot{x}+i \dot{y}, \dot{w}) \in T_{q}(\tilde{M})$ at $q=(x+i y, w) \in \tilde{M}$. Then $\dot{x}=$ $h_{y} \dot{y}+h_{w} \dot{w}+h_{\bar{w}} \overline{\dot{w}}+h_{t} \dot{t}$, where the partial derivatives $h_{y}, \ldots$ are evaluated at $(y, w, t)$ such that $x=h(y, w, t)$. We call $\dot{t}$ the $t$-component of $v$.

Thus, the $y$-component of $\dot{A}$ must satisfy the linearized Bishop's equation

$$
\dot{y}=T_{1}\left(h_{y} \dot{y}+h_{w} \dot{w}+h_{\bar{w}} \overline{\dot{w}}+h_{t} \dot{\tau}\right),
$$

where $\dot{\tau}$ is the $t$-component of $\left.\dot{A}\right|_{b \Delta}$. The partial derivatives $h_{y}, \ldots$ are evaluated at $(y(\zeta), w(\zeta), \tau(\zeta))$, where $\tau$ is the $t$-component of $\left.A\right|_{b \Delta}$.

We restrict to deformations $\dot{A}$ with the $w$ - and $t$-components $\zeta \mapsto \dot{w}(\zeta)$ and $\zeta \mapsto \dot{\tau}(\zeta)$ in $C^{k, \alpha}$. In this case the equation (2.8) has a unique solution $\zeta \mapsto \dot{y}(\zeta)$ in $C^{k-1, \alpha}$. Thus, the set of deformations of $A \in \tilde{\mathcal{A}}_{p}$ is identified with $\tilde{\mathcal{B}}$. We write $\dot{A} \leftrightarrow(\dot{w}, \dot{\tau}) \in \tilde{B}$.

Following [T1], we associate to a disc $A \in \tilde{\mathcal{A}}_{p}$, a $C^{k-1, \alpha}$ real $m \times m$ matrix function $\zeta \mapsto G(\zeta)$ on $b \Delta$ as the unique solution to the equation

$$
G=\mathbf{1}-T_{1}\left(G h_{y}\right),
$$

where $h_{y}$ is evaluated at $(y(\zeta), w(\zeta), \tau(\zeta))$ as in (2.8) and $\mathbf{1}$ denotes the identity matrix. Since $A$ is small, $G$ is nondegenerate.

We introduce the following notation. For a $C^{1, \alpha}$ function $\phi$ on the unit circle with $\phi(1)=0$, we write

$$
\mathcal{J}(\phi)=\frac{1}{\pi} \int_{0}^{2 \pi} \frac{\phi\left(e^{i \theta}\right) d \theta}{\left|e^{i \theta}-1\right|^{2}}=\frac{i}{\pi} \int_{b \Delta} \frac{\phi(\zeta) d \zeta}{(\zeta-1)^{2}},
$$

where the integral is understood in the sense of principal value. Note that for any function $\phi \in C^{1, \alpha}(\bar{\Delta})$ holomorphic in $\Delta$ with $\phi(1)=0$, we have

$$
\mathcal{J}(\phi)=-\frac{\partial \phi(1)}{\partial \zeta}
$$

Lemma 2.3. The solution of (2.8) has the form

$$
\dot{y}=\left(\mathbf{1}+h_{y}^{2}\right)^{-1}\left(G^{-1} T_{1}(G \phi)-h_{y} \phi\right),
$$

where $\phi=h_{w} \dot{w}+h_{\bar{w}} \overline{\dot{w}}+h_{t} \dot{\tau}$. Moreover

$$
\mathcal{J}(\dot{x})=\mathcal{J}(G \phi),
$$

where $\dot{x}$ is the $x$-component of $\dot{A}$.

The proof of this lemma is given in [T1]. 


\section{Defect of discs and proof of (A)}

Let $M$ and $M_{0}$ be the same as in Section 2. In addition, assume $k \geq 2$. We define the defect of a disc $A \in \tilde{\mathcal{A}}_{p}$.

Let $\mathbf{R}^{m *}$ be the dual space to $\mathbf{R}^{m}$. To apply the matrix notation, we regard it as the space of row $m$-vectors. Let $A \in \tilde{\mathcal{A}}_{p}$. We set

$V^{*}(A)=\left\{c \in \mathbf{R}^{m *}: c G h_{w}\right.$ extends holomorphically to $\left.\Delta, c G h_{t}=0\right\}$,

$\operatorname{def}(A)=\operatorname{dim}\left(V^{*}(A)\right)$,

where $h_{w}$ and $h_{t}$ are evaluated at $(y(\zeta), w(\zeta), \tau(\zeta))$ as in (2.8). Note that $\operatorname{rank}\left(G h_{t}\right)=\operatorname{rank}\left(h_{t}\right)=l$. Therefore, $\operatorname{def}(A) \leq m-l=\operatorname{codim}(M)$. One can see that $\operatorname{def}(A)$ is the same as the defect of $A$ as a disc attached to $\tilde{M}$ (see $[\mathrm{T} 1]$ and $[\mathrm{BRT}])$. We set

$$
V^{\prime}(A)=\operatorname{Span}\left\{-\partial \dot{A}(1) / \partial \zeta \bmod T_{p}\left(M_{0}\right): \dot{\tau}(\zeta) \in \Gamma \cup 0 \text { for } \zeta \in b \Delta\right\}
$$

where $\dot{A}$ denotes a deformation of $A$ and $\dot{\tau}$ is the $t$-component of $\dot{A}$.

Lemma 3.1. For $A \in \mathcal{A}_{p}, \operatorname{dim}\left(V^{\prime}(A)\right)=m-\operatorname{def}(A)$.

Proof. The proof is very similar to that of a related result of [T1]. Since $T_{p}\left(M_{0}\right)$ is given by the equation $x=0$, the normal space $T_{p}\left(\mathbf{C}^{N}\right) / T_{p}\left(M_{0}\right)$ is identified with the $x$-space $\mathbf{R}^{m}$. We show that $V^{*}(A)=V^{\prime}(A)^{\perp}$.

Let $c \in V^{\prime}(A)^{\perp}$. Let $\dot{A}=(\dot{x}+i \dot{y}, \dot{w})$ be a deformation of $A$ with the $t$-component $\dot{\tau}$ taking values in $\Gamma \cup 0$. By (2.11), the $x$-component of $-\partial \dot{A}(1) / \partial \zeta$ is $\mathcal{J}(\dot{x})$. Thus, $c \mathcal{J}(\dot{x})=0$. By $(2.13)$,

$$
0=c \mathcal{J}(\dot{x})=\mathcal{J}\left(c G h_{w} \dot{w}\right)+\mathcal{J}\left(c G h_{\bar{w}} \overline{\dot{w}}\right)+\mathcal{J}\left(c G h_{t} \dot{\tau}\right)
$$

This holds for every pair $(\dot{w}, \dot{\tau}) \in \mathcal{B}(2.7)$, in particular $(\dot{w}, 0),(i \dot{w}, 0)$ and $(0, \dot{\tau})$. Therefore,

$$
\mathcal{J}\left(c G h_{w} \dot{w}\right)=0, \quad \mathcal{J}\left(c G h_{t} \dot{\tau}\right)=0 .
$$

Using the holomorphic form of $\mathcal{J}$ in (2.10), we see that $c G h_{w}$ must extend holomorphically to $\Delta$. Since $\dot{\tau}$ is any function with $\dot{\tau}(\zeta) \in \Gamma \cup 0$ and $\dot{\tau}(1)=0$, we conclude that $c G h_{t}=0$ identically. Hence $c \in V^{*}(A)$. The converse is obvious. 
Lemma 3.2. For $A \in \mathcal{A}_{p}, V^{\prime}(A) \subset V_{p}^{\prime}$.

Proof. The proof is a simple check of the definitions (2.2) and (3.2). Indeed, let $\dot{A}$ be a deformation of $A$ with the $t$-component $\dot{\tau}$ with $\dot{\tau}(\zeta) \in \Gamma \cup 0$. We need to show that $-\partial \dot{A}(1) / \partial \zeta \bmod T_{p}\left(M_{0}\right) \in V_{p}^{\prime}$.

Let $w$ and $\tau$ be the $w$ - and $t$-components of $A$, and let $\dot{w}$ and $\dot{\tau}$ be the $w$ - and $t$-components of $\dot{A}$. Using Proposition 2.2, we construct the family of $\operatorname{discs} \zeta \mapsto A(\zeta, s)$ (where $s>0$ ), having the $w$-and $t$-components $w+s \dot{w}$ and $\tau+s \dot{\tau}$. Since $\Gamma$ is convex, $\tau(\zeta), \dot{\tau}(\zeta) \in \Gamma \cup 0$ and $s>0$, the discs belong to $\mathcal{A}_{p}$. Then $\dot{A}(\zeta)=\left.\frac{d}{d s}\right|_{s=0} A(\zeta, s)$. Therefore,

$$
-\partial \dot{A}(1) / \partial \zeta \bmod T_{p}\left(M_{0}\right)=\left.\frac{d}{d s}\right|_{s=0}\left(-\partial A(1, s) / \partial \zeta \bmod T_{p}\left(M_{0}\right)\right) .
$$

Since the expression in parentheses is in $V_{p}^{\prime}$, the derivative is also in $V_{p}^{\prime}$, which is what we need. (We use the fact that $M$ is $C^{k, \alpha}$ with $k \geq 2$.)

We now define the defect of $M$ at $p$.

$$
\operatorname{def}\left(p, M_{0}, M\right)=\liminf \operatorname{def}(A) \quad \text { as } A \in \mathcal{A}_{p}, \quad\|A\|_{C^{k, \alpha}} \rightarrow 0 .
$$

End of the proof of (A). Let $d=\operatorname{def}\left(p, M_{0}, M\right)$. Then there exists $A \in \mathcal{A}_{p}$ with $\operatorname{def}(A)=d$. By Lemmas 3.1 and $3.2, \operatorname{dim}\left(V_{p}^{\prime}\right) \geq m-d$. The statement (A) now follows by Proposition 2.1 .

\section{Proof of (B)}

Let $M, M_{0}$ and $p$ be the same as in the previous section. We construct the submanifold $S$ as a union of boundaries of analytic discs attached to $M \cup M_{0}$. We consider the evaluation map

$$
\mathcal{F}: \tilde{\mathcal{A}}_{p} \rightarrow \tilde{M}, \quad \mathcal{F}: A \mapsto A(-1) .
$$

Since we identify $\tilde{\mathcal{A}}_{p}$ with a neighborhood of zero in $\tilde{B}(2.6)$, we also regard $\mathcal{F}$ as a mapping on this neighborhood. According to results on the regularity of Bishop's equations [T2] [T4], $\mathcal{F}$ is $C^{k, \beta}$ for every $0<\beta<\alpha$.

Let $A \in \tilde{\mathcal{A}}_{p}, q=\mathcal{F}(A)=A(-1)$. Let $L(A)=\mathcal{F}^{\prime}(A) \tilde{\mathcal{B}} \subset T_{q}(\tilde{M})$, the image of the derivative of $\mathcal{F}$ at $A$.

\section{Proposition 4.1.}

(i) $\operatorname{dim} L(A)=\operatorname{dim}(M)-\operatorname{def}(A)$;

(ii) $T_{q}^{c}(\tilde{M}) \subset L(A)$. 
Proof. To prove (i), we construct an isomorphism between $V^{*}(A)(3.1)$ and $L(A)^{\perp} \subset T_{q}^{*}(\tilde{M})$. To prove (ii), we show that $L(A)^{\perp}$ annihilates $T_{q}^{c}(\tilde{M})$.

Let $\omega=P d y+Q d w+\bar{Q} d \bar{w}+R d t \in L(A)^{\perp}$. This means precisely that for every deformation $\dot{A} \leftrightarrow(\dot{w}, \dot{\tau}) \in \tilde{\mathcal{B}}$,

$$
(P \dot{y}+Q \dot{w}+\bar{Q} \overline{\dot{w}}+R \dot{\tau})(-1)=0
$$

where $\dot{y}$ is the $y$-component of $\dot{A}$. Using (2.12), we get

$$
\left(P\left(\mathbf{1}+h_{y}^{2}\right)^{-1}\left(G^{-1} T_{1}(G \phi)-h_{y} \phi\right)+Q \dot{w}+\bar{Q} \overline{\dot{w}}+R \dot{\tau}\right)(-1)=0,
$$

where $\phi=h_{w} \dot{w}+h_{\bar{w}} \overline{\dot{w}}+h_{t} \dot{\tau}$. This holds for every pair $(\dot{w}, \dot{\tau}) \in \tilde{\mathcal{B}}$, in particular, $(\dot{w}, 0),(i \dot{w}, 0)$ and $(0, \dot{\tau})$. Therefore,

$$
\begin{aligned}
& \left(P\left(\mathbf{1}+h_{y}^{2}\right)^{-1}\left(G^{-1} T_{1}\left(G h_{w} \dot{w}\right)-h_{y} h_{w} \dot{w}\right)+Q \dot{w}\right)(-1)=0, \\
& \left(P\left(\mathbf{1}+h_{y}^{2}\right)^{-1}\left(G^{-1} T_{1}\left(G h_{t} \dot{\tau}\right)-h_{y} h_{t} \dot{\tau}\right)+R \dot{\tau}\right)(-1)=0 .
\end{aligned}
$$

Note that for every $\psi \in C^{0, \alpha}(b \Delta)$,

$$
\left(T_{1} \psi\right)(-1)=\frac{2}{\pi} \int_{b \Delta} \frac{\psi(\zeta) d \zeta}{\zeta^{2}-1} .
$$

Let $\dot{w}(-1)=0$ and $\dot{\tau}(-1)=0$. Then (4.2) yields

$$
\int_{b \Delta} c G h_{w} \frac{\dot{w}(\zeta)}{\zeta^{2}-1} d \zeta=0, \quad \int_{b \Delta} c G h_{t} \frac{\dot{\tau}(\zeta)}{\zeta^{2}-1} d \zeta=0
$$

where

$$
c=\left(P\left(\mathbf{1}+h_{y}^{2}\right)^{-1} G^{-1}\right)(-1) .
$$

Since (4.3) holds for every $(\dot{w}, \dot{\tau}) \in \tilde{\mathcal{B}}$ vanishing at $-1, c G h_{w}$ must extend holomorphically to $\Delta$, and $c G h_{t}$ must vanish identically. Hence $c \in V^{*}(A)$.

Since $c G h_{w} \dot{w}$ is holomorphic and $\dot{w}(1)=0$, we have $T_{1}\left(c G h_{w} \dot{w}\right)=$ $-i c G h_{w} \dot{w}$. Plugging this and $c G h_{t}=0$ in (4.2), we get

$$
\begin{aligned}
& \left(P\left(\left(h_{y}-i \mathbf{1}\right)^{-1} h_{w}-Q\right) \dot{w}(-1)=0,\right. \\
& \left(P\left(\left(\mathbf{1}+h_{y}^{2}\right)^{-1} h_{y} h_{t}-R\right) \dot{\tau}(-1)=0 .\right.
\end{aligned}
$$

Since $\dot{w}(-1) \in \mathbf{C}^{n}$ and $\dot{\tau}(-1) \in \mathbf{R}^{l}$ are arbitrary,

$$
Q=P\left(h_{y}-i \mathbf{1}\right)^{-1} h_{w}, \quad R=P\left(\mathbf{1}+h_{y}^{2}\right)^{-1} h_{y} h_{t},
$$


where $h_{y} \ldots$ are evaluated at $q$. A simple check shows that (4.6) means precisely that $\omega \in T_{q}^{c}(\tilde{M})^{\perp}$, which proves (ii).

We claim that the mapping $\omega \mapsto c$ given by (4.4) is an isomorphism. Indeed, it is linear by (4.4). It is injective because $Q$ and $R$ are uniquely defined by $P$. It is also surjective, because, given $c \in V^{*}(A)$, we can use (4.4) and (4.6) to get $\omega \in L(A)^{\perp}$, which completes the proof of (i). The proposition is now proved.

We fix a smooth scalar function $\psi$ on $b \Delta$ such that $\psi(\zeta)>0$ for every $\zeta \in b \Delta$ except that $\psi(1)=0$. We take $\psi$ with $\psi(-1)=1$. For $\epsilon>0$ we introduce the subspace

$(4.7)$

$\mathcal{B}_{\epsilon}=\left\{(w, \tau) \in \tilde{\mathcal{B}}: \tau=\lambda \psi+\phi, \lambda \in \mathbf{R}^{l}, \phi(\zeta)=0\right.$ if $|\zeta+1|<\epsilon$ or $\left.|\zeta-1|<\epsilon\right\}$.

We set $L_{\epsilon}(A)=\mathcal{F}^{\prime}(A)\left(\mathcal{B}_{\epsilon}\right)$.

Lemma 4.2. For every $A \in \mathcal{A}_{p}$ there exists $\epsilon>0$ such that $L_{\epsilon}(A)=L(A)$.

Proof. Since $L_{\epsilon}(A)$ and $L(A)$ are finite dimensional, it suffices to show that $\bigcup_{\epsilon>0} L_{\epsilon}(A)=L(A)$. By passage to orthogonal complements in $T_{q}^{*}(M)$, it reduces to $\bigcap_{\epsilon>0} L_{\epsilon}(A)^{\perp}=L(A)^{\perp}$.

Using the same notation as in the proof of Proposition 4.1, let $\omega \in$ $\bigcap_{\epsilon>0} L_{\epsilon}(A)^{\perp}$. We then find that (4.3) holds for all $\dot{\tau}$ vanishing near \pm 1 . This suffices to conclude that $c$ given by (4.4) is in $V^{*}(A)$. We further get that since $\dot{\tau}(-1)=\lambda \psi(-1) \in \mathbf{R}^{l}$ is arbitrary, (4.5) still implies (4.6). Thus $\omega \in L(A)^{\perp}$.

We now turn to the proof of (B). Let $d=\operatorname{def}\left(p, M_{0}, M\right)$ defined by (3.3). Let $A_{0} \in \mathcal{A}_{p}$ be a disc with $\operatorname{def}\left(A_{0}\right)=d$. Let $A_{0} \leftrightarrow\left(w_{0}, \tau_{0}\right)$. We can assume that $A_{0}(-1)=p=0$, otherwise we replace $A_{0}$ by the disc $\zeta \mapsto A_{0}\left(\zeta^{2}\right)$, which has the same defect and is still small in $C^{k, \alpha}$. We can further assume that $\zeta= \pm 1$ are the only points on $b \Delta$ where $A_{0}(\zeta) \in M_{0}$ because small perturbations can only reduce the defect of $A_{0}$, which is already of minimum defect among sufficiently small discs in $\mathcal{A}_{p}$.

We fix $\epsilon>0$ such that $L_{\epsilon}\left(A_{0}\right)=L\left(A_{0}\right)$. We choose $\dot{A}_{j} \leftrightarrow\left(\dot{w}_{j}, \dot{\tau}_{j}\right) \in \mathcal{B}_{\epsilon}$, $1 \leq j \leq r=\operatorname{dim}(M)-d$, such that $\mathcal{F}^{\prime}\left(A_{0}\right) \dot{A}_{j}$ form a basis in $L_{\epsilon}\left(A_{0}\right)$ whence in $L\left(A_{0}\right)$. Specifically, we can take $\dot{w}_{j}=0, \dot{\tau}_{j}=e_{j} \psi$ for $1 \leq j \leq l$, where $e_{j}$ is the unit vector of the $j$-th coordinate in $\mathbf{R}^{r}$. Indeed, these $\dot{A}_{j}$, $1 \leq j \leq l$, have linearly independent $t$-components. We can further assume that

$$
\dot{\tau}_{j}(\zeta)=0 \quad \text { if }|\zeta-1|<\epsilon \text { or }|\zeta+1|<\epsilon \quad \text { for } l<j \leq r
$$


(Otherwise, for $j>l$, we replace $\dot{A}_{j}$ by $\dot{A}_{j}-\sum_{\nu=1}^{l} u_{\nu} \dot{A}_{\nu}$, where $\left(u_{1}, \ldots, u_{l}\right)^{T}$ $=\dot{\tau}_{j}(-1)$, see $(4.7)$.)

We restrict the evaluation map to the $\operatorname{discs} A_{s} \in \tilde{\mathcal{A}}_{p}, s \in \mathbf{R}^{r}, A_{s} \leftrightarrow$ $\left(w_{s}, \tau_{s}\right)$, where

$$
\begin{aligned}
w_{s} & =w_{0}+\sum_{j=1}^{r} s_{j} \dot{w}_{j} \quad \text { and } \\
\tau_{s} & =\tau_{0}+\sum_{j=1}^{r} s_{j} \dot{\tau}_{j}=\tau_{0}+\sum_{j=1}^{l} s_{j} e_{j} \psi+\sum_{j=l+1}^{r} s_{j} \dot{\tau}_{j}
\end{aligned}
$$

Let $H: s \mapsto \mathcal{F}\left(A_{s}\right)=A_{s}(-1)$. The mapping $H$ is $C^{k, \beta}$ in a neighborhood of zero $U \subset \mathbf{R}^{r}$ for all $0<\beta<\alpha$. Since $\partial H(0) / \partial s_{j}=\mathcal{F}^{\prime}\left(A_{0}\right) \dot{A}_{j}$ are linearly independent, $H$ is an immersion if $U$ is small.

Let $\tilde{S}=H(U)$. Since $\tau_{s}(-1)=\left(s_{1}, \ldots, s_{l}\right)^{T}$, we have $\left(s_{1}, \ldots, s_{l}\right)^{T} \in \Gamma$ iff $H(s) \in M$. Therefore, $S=\tilde{S} \cap M=H\left(\left(\Gamma \times \mathbf{R}^{r-l}\right) \cap U\right)$ is a manifold with edge $S_{0}=\tilde{S} \cap M_{0}=H\left(\left(0 \times \mathbf{R}^{r-l}\right) \cap U\right)$.

We now prove that $T^{c}(S)=\left.T^{c}(M)\right|_{S}$. We first note that if $s \in \mathbf{R}^{r}$ is small, then $\left(s_{1}, \ldots, s_{l}\right)^{T}=\tau_{s}(-1) \in \Gamma$ implies that $\tau_{s}(\zeta) \in \Gamma$ for all $\zeta \in b \Delta$, $\zeta \neq 1$. Indeed, since $\Gamma$ is convex and $\psi(\zeta) \geq 0$, by (4.8) the right-hand side of (4.9) is in $\Gamma$ for $\zeta$ close to \pm 1 . If $\zeta$ is outside a neighborhood of \pm 1 , $\tau_{s}(\zeta) \in \Gamma$ holds for small $s$ because $\tau_{0}(\zeta) \in \Gamma$ for $\zeta \in b \Delta, \zeta \neq \pm 1$.

Let $q \in S, q=H(s), s \in\left(\Gamma \times \mathbf{R}^{r-l}\right) \cap U$. By the remark above, $A_{s} \in \mathcal{A}_{p}$, that is $A_{s}$ is attached to $M \cap M_{0}$. Since $H$ is obtained as a restriction of the evaluation map $\mathcal{F}$, we have $T_{q}(S) \subset L\left(A_{s}\right)$. Since $A_{s} \in \mathcal{A}_{p}$ is close to $A_{0}$, $\operatorname{def}\left(A_{s}\right)=d$. Therefore, by Proposition $4.1, \operatorname{dim} T_{q}(S)=r=\operatorname{dim}(M)-d=$ $\operatorname{dim} L\left(A_{s}\right)$. Hence, $T_{q}(S)=L\left(A_{s}\right) \supset T_{q}^{c}(M)$, which is what we need.

The proof of $(\mathrm{B})$ is complete.

\section{References}

[A] R. A. Ayrapetian, Extension of $C R$ functions from piecewise smooth $C R$ manifolds, Mat. Sbornik 134 (1987), 111-120.

[BR] M. S. Baouendi and L. Rothschild, Cauchy-Riemann functions on manifolds of higher codimension in complex space, Invent. Math. 101 (1990), 45-56.

[BRT] M. S. Baouendi, L. Rothschild and J. M. Trépreau, On the geometry of analytic discs attached to real manifolds, J. Diff. Geom. 39 (1994), 379-405.

[BT] M. S. Baouendi and F. Treves, A property of the functions and distributions annihilated by a locally integrable system of complex vector fields, Ann. of Math. 114 (1981), 387-421.

[B] E. Bishop, Differentiable manifolds in complex Euclidean space, Duke Math. J. 32 (1965), 1-21. 
[Bo] A. Boggess, CR manifolds and the tangential Cauchy-Riemann complex, CRC Press, Boca Raton, FL, 1991.

[BP] A. Boggess and J. T. Pitts, CR extension near a point of higher type, Duke Math. J. 52 (1985), 67-102.

[T1] A. E. Tumanov, Extending CR functions on a manifold of finite type over a wedge, Mat. Sbornik 136 (1988), 129-140.

[T2] _ Extending CR functions into a wedge, Mat. Sbornik 181 (1990), 951964.

[T3] Connections and propagation of analyticity for CR functions, Duke Math. J. 73 (1994), 1-24.

[T4] On the propagation of extendibility of CR functions, Complex analysis and geometry, Lect. Notes in Pure and Appl. Math., Dekker, to appear.

Department of M athematics, Univerity of Illinois, URbana IL 61801

E-mail address: tumanov@math.uiuc.edu 